\title{
Comparison and experimental realization of different phase- only grating designs and optimal triplicators
}

\section{Comparación y realización experimental de diferentes diseños de redes de fase y triplicadores óptimos}

\author{
F. Aroca, I. Morenos, ${ }^{\text {* }}$ \\ Departamento de Ciencia de Materiales, Óptica y Tecnología Electrónica, \\ Universidad Miguel Hernández de Elche, 03202 Elche (Alicante), Spain \\ (*) E-mail: i.moreno@umh.es S: SEDOPTICA member \\ Received: 21/04/2016 Accepted: 19/07/2016 \\ DOI: $10.7149 /$ OPA.49.3.49007
}

\begin{abstract}
:
In this work we present the experimental realization of a laser multiple beam splitter based phaseonly gratings displayed onto a spatial light modulator. We compare the performance binary phaseonly Dammann gratings with an optimal phase triplicator. Experimental results are included, obtained with a liquid crystal on silicon (LCOS) display.
\end{abstract}

Key words: Diffraction gratings, Phase-only modulators.

\section{RESUMEN:}

Este trabajo presenta la realización experimental de un divisor de haz laser basado en redes de difracción implementadas en un modulador puro de fase. Comparamos las prestaciones de redes binarias de fase de Dammann, con el diseño del triplicador óptimo de fase. Los resultados experimentales se han realizado con un modulador del tipo LCOS - Liquid Crystal on Silicon.

Palabras clave: Redes de difracción, Moduladores puros de fase.

\section{REFERENCES AND LINKS / REFERENCIAS Y ENLACES}

[1] A. W. Lohmann, "A pre-history of computer-generated holography", Optics \& Photonics News 19, 2641 (2008). http://dx.doi.org/10.1364/opn.19.2.000036

[2] H. P. Herzig, Edt., Micro-Optics. Elements, Systems and Applications, Taylor \& Francis (1997).

[3] I. Moreno, A. Márquez, J. A. Davis, J. Campos, M. J. Yzuel, “Realización de elementos ópticos difractivos de amplitud compleja mediante moduladores de cristal líquido optimizados", Opt. Pura Apl. 38, 1-12 (2005).

[4] J. A. Davis, I. Moreno, “Generation of Laser Beams by Digital Holograms”, Chapter 6 in Laser Beam Propagation: Generation and Propagation of Customized Light, A. Forbes Editor, CRC Press, Taylor \& Francis (2014). http://dx.doi.org/10.1201/b16548-9

[5] J. Cheng, C. Gu, D. Zhang, S.-C. Chen, "High-speed femtosecond laser beam shaping based on binary holography using a digital micromirror device”, Opt. Lett. 40, 4875-4879 (2015). http://dx.doi.org/10.1364/OL.40.004875

[6] J. W. Goodman, Introduction to Fourier Optics, 2ª edición, McGraw-Hill, New York (1996).

[7] H. Dammann, K. Görtler, "High-efficiency in-line multiple imaging by means of multiple phase holograms", Optics Commun. 3, 312-315 (1971). http://dx.doi.org/10.1016/0030-4018(71)90095-2 
[8] H. Dammann, E. Klotz, “Coherent optical generation and inspection of two-dimensional periodic structures”, Opt. Acta 24, 505-515 (1977). http://dx.doi.org/10.1080/713819570

[9] C. Zhou, L. Liu, "Numerical study of Dammann array illuminators”, Appl. Opt. 34, 5961-5969 (1995). http://dx.doi.org/10.1364/A0.34.005961

[10] I. Moreno, J. A. Davis, D. M. Cottrell, N. Zhang, X.-C. Yuan, "Encoding generalized phase functions on Dammann gratings", Opt. Lett. 35, 1536-1538 (2010). http://dx.doi.org/10.1364/OL.35.001536

[11] F. Gori, M. Santarsiero, S. Vicalvi, R. Borghi, G. Cincotti, E. di Fabrizio, M. Gentili, “Analytical derivation of the optimum triplicator", Opt. Commun. 157, 13-16 (1998). http://dx.doi.org/10.1016/S0030-4018(98)00518-5

[12] I. Moreno, C. Lemmi, A. Márquez, J. Campos, y M. J. Yzuel, “Modulation light efficiency of diffractive lenses displayed in a restricted phase-mostly modulation display”, Appl. Opt. 43, 6278-6284 (2004). http://dx.doi.org/10.1364/A0.43.006278

[13] F. Aroca, "Diseño y realización de redes de Dammann programables", Proyecto Final de Carrera, Ingeniería Industrial, Universidad Miguel Hernández de Elche (2015).

[14] Z. Zhang, G. Lu, F.T.S. Yu, "Simple method for measuring phase modulation in liquid crystal televisions”, Opt. Eng. 33, 3018-3022 (1994). http://dx.doi.org/10.1117/12.177518

[15] F. J. Salgado-Remacha, "Moiré patterns with color LCD displays", Opt. Pura Apl. 49, 51-55 (2016). http://dx.doi.org/10.7149/OPA.49.1.51

\section{Introducción}

La invención del láser y de la holografía en los años sesenta del siglo XX [1], y los avances en las técnicas de microfabricación y replicación [2] ha proporcionado las herramientas para el desarrollo de la Óptica Difractiva. Los elementos ópticos difractivos (DOE, Diffractive Optical Elements) se diseñan y aplican hoy en día en multitud de instrumentos y aparatos. De igual manera, en paralelo, el desarrollo en las últimas décadas de los modernos dispositivos moduladores espaciales de luz (SLM, Spatial Light Modulators), con resolución espacial suficiente para reproducir DOEs dinámicos, esto es, elementos difractivos que pueden ser programados y controlados en tiempo real desde un ordenador. Las dos tecnologías principales de SLMs son los dispositivos de cristal líquido (LCD, Liquid Crystal Displays), y los dispositivos de espejos deformables (DMD, Digital Micromirror Device). Los LCD tienen la ventaja para aplicaciones difractivas de producir directamente variaciones de fase $[3,4]$. Los DMD comerciales son dispositivos moduladores binarios de intensidad, por lo que solamente pueden reproducir DOEs de amplitud. Por el contrario, los DMD son dispositivos mucho más veloces que los LCD [5].

Este trabajo se centra en la realización y análisis de redes de difracción puras de fase, elementos que podemos considerar como los DOEs más sencillos. La transmisión del haz de luz por la red provoca la generación de diferentes haces difractados. Los factores que determinan las características de estos órdenes de difracción son la longitud de onda de la luz incidente, el periodo de la red, y el perfil de transmisión/reflexión de la red. La teoría de la Óptica de Fourier interpreta los órdenes de difracción como los armónicos de la frecuencia fundamental de la red [6]. Esta aproximación es válida siempre que nos limitemos al ámbito de la aproximación paraxial, y consideremos periodos de la red de difracción suficientemente grandes en comparación con la longitud de onda de la luz. En este trabajo adoptamos esta aproximación.

En general, las redes de difracción producen órdenes con diferente intensidad. En los años 70, H. Dammann desarrolló un método matemático para generar redes que producen órdenes de difracción con igual intensidad $[7,8]$. Su método se basó en diseñar redes de fase binarias con transiciones de fase $0-\pi$ y $\pi-0$ en el perfil periódico de la red, calculadas para igualar la intensidad de un número dado de armónicos. Con el desarrollo de modernos métodos de micro-óptica, las redes de Dammann pasaron a ser un elemento popular [9], y recientemente se han realizado también en dispositivos SLM binarios de fase [10].

No obstante, la posibilidad de realizar no solamente perfiles binarios, sino también perfiles continuos de fase, ha propiciado el desarrollo de diseños que permiten generar redes de difracción con órdenes de igual intensidad, pero con mayor eficiencia que la que proporcionan las redes binarias de fase de Dammann. En este trabajo, en particular, se ha empleado el diseño del triplicador óptimo presentado por Gori et al. en [11]. Ésta es una red de difracción pura de fase que genera tres órdenes simétricos, el orden 0 y los 
órdenes \pm 1 , con idéntica intensidad y con la máxima eficiencia posible. La derivación de las componentes armónicas en función del perfil de modulación del dispositivo SLM permite además unificar el diseño de la red y los efectos de modulación en el cálculo de la intensidad de los órdenes de difracción [12].

El trabajo que se presenta supone un interesante ejercicio práctico, útil para la introducción de estos aspectos de la Óptica, tanto a nivel educativo como de iniciación a la investigación en el campo de la óptica difractiva con dispositivos SLM. Este trabajo se ha realizado en el marco de un proyecto final de carrera en la Universidad Miguel Hernández de Elche [13].

El trabajo se estructura en las siguientes secciones: tras esta introducción, la sección 2 presenta un breve resumen de las propiedades de las redes de fase, que incluye redes de fase lineal, redes de fase binaria, redes de Dammann y el triplicador óptimo de Gori. A continuación, la sección 3 presenta y describe el sistema experimental construido para su evaluación en un SLM de tipo LCOS. La sección 4 presenta los resultados experimentales obtenidos. Y finalmente la sección 5 presenta las conclusiones del trabajo.

\section{Diseño de redes de fase}

En esta sección se describen los diversos diseños de redes de fase que hemos evaluado mediante el SLM. Suponiendo la aproximación escalar y paraxial de la Óptica de Fourier, trataremos el patrón de órdenes de difracción generados como la transformada de Fourier de la función de transmisión de las redes. En esta aproximación, la función $g(x)$ que describe la transmisión/reflexión de red de difracción puede desarrollarse como una serie de Fourier, esto es una descomposición infinita de ondas planas con diferente inclinación, en la forma:

$$
g(x)=\sum_{n=-\infty}^{+\infty} G_{n} \exp (\operatorname{in} 2 \pi x / p)
$$

En esta ecuación $p$ indica el periodo de la red y $G_{n}$ son los coeficientes del desarrollo de Fourier asociado a la forma específica de la función $g(x)$ que describe la transmisión de la red. Cada una de las exponenciales de la expresión anterior corresponde a una onda plana que genera un orden de difracción.

La transformada de Fourier $G(v)$ de $g(x)$ adopta la forma siguiente:

$$
G(v)=\sum_{n=-\infty}^{+\infty} G_{n} \delta\left(v-\frac{n}{p}\right)
$$

donde $v$ indica la frecuencia espacial, y donde cada función $\delta$ corresponde a un orden de difracción. La intensidad relativa de cada orden de difracción viene dada por el módulo al cuadrado del correspondiente coeficiente del desarrollo de Fourier:

$$
I_{n}=\left|G_{n}\right|^{2}
$$

Dependiendo del perfil de transmisión de la red se obtienen distintos patrones de difracción. En este trabajo se consideran redes de difracción puras de fase, esto es, la función de transmisión/reflexión es del tipo $g(x)=\exp (i \phi(x))$. A continuación, se resumen brevemente los tres tipos de perfil de redes que hemos aplicado: (a) perfil de fase lineal, (b) perfil de fase binario, y (c) perfil de fase del triplicador óptimo.

\section{2.a. Redes de perfil de fase lineal}

Este perfil se expresa matemáticamente como:

$$
g(x)=\exp (\operatorname{in} 2 \pi x / p) .
$$

Se trata de una red pura de fase con un perfil lineal que alcanza una variación total de la fase de $2 \pi$ radianes en cada periodo, tal y como indica la figura 1(a). Esta red difracta el haz incidente completamente al primer orden de difracción $(n=1)$, esto es genera únicamente el armónico principal. En la ecuación (1) solamente hay un término, y las intensidades relativas de los órdenes de difracción son $I_{1}=100 \%$, e $I_{n \neq 1}=0$.

Si la variación de fase no se ajusta al valor $2 \pi$ (figura $1(b)$ ), la función de transmisión de la red de fase lineal puede describirse matemáticamente como [12]:

$$
g(x)=\left[\exp \left(i \frac{2 \pi(1-c) x}{p}\right) \cdot \operatorname{rect}\left(\frac{x}{p}\right)\right] \otimes \sum_{n=-\infty}^{+\infty} \delta(x-n p),
$$


donde el símbolo $\otimes$ indica la operación de convolución, y donde $c$ es un parámetro que define la máxima variación de fase en la red. Si $c \neq 0$ se generan otros órdenes de difracción (otras componentes armónicas), y la intensidad relativa del orden $n$ viene dada por la relación [11]:

$$
I_{n}=\operatorname{sinc}^{2}(1-c-n),
$$

donde la función seno-cociente se define $\operatorname{como} \operatorname{sinc}(x)=\sin (\pi x) /(\pi x)$. En el caso particular en que la máxima modulación de fase es de solamente $\pi$ radianes $(c=0,5)$, los órdenes $n=0$ y $n=+1$ tienen la misma intensidad relativa, cada uno de ellos con un factor $I_{0,+1}=40,5 \%$ de la energía total. Esta red puede considerarse por tanto un duplicador óptico $(0,+1)$ con una eficiencia total del $\eta=81 \%$ (el $19 \%$ de le energía restante se reparte en otros órdenes de difracción distintos de $n=0$ y $n=+1$ ).
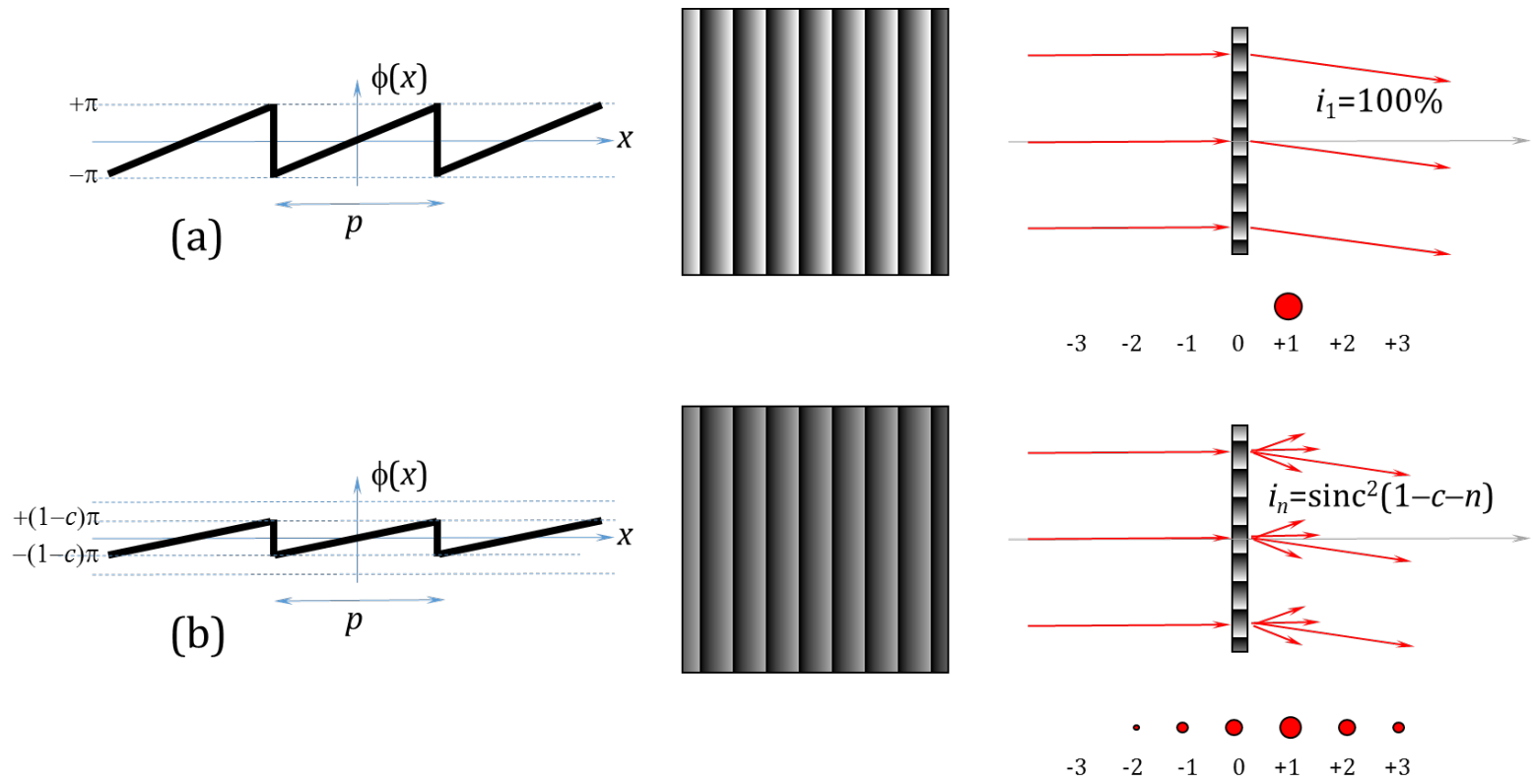

Fig.1. Ilustración de la red de fase lineal (a) con una variación de fase $2 \pi$, (b) con una profundidad de fase arbitraria $2 \pi(1-c)$.

\section{2.b. Redes de fase de perfil binario}

Las redes de difracción binarias pueden interpretarse como una modificación del perfil de fase de la red de fase lineal. La figura 2 ilustra esta modificación: se parte de la red de fase lineal ideal, pero se aplica una tabla de conversión (LUT, Look-Up Table) de los valores de fase que genera la red de fase binaria. Valores de fase positivos toman el valor $+\phi / 2$, mientras que valores de fase negativos adoptan el valor $-\phi / 2$, de modo que hay una diferencia de fase $\phi$ entre los dos niveles de la red. El resultado es la generación de órdenes de difracción diferentes a $n=1$, correspondientes a la generación de otros armónicos de la frecuencia fundamental de la red.

Esta interpretación de la modificación del perfil de fase de la red resulta interesante pues permite extender los conceptos de generación de diferentes componentes armónicas a otros elementos difractivos, como por ejemplo las lentes de Fresnel [12], o redes difractivas que producen vórtices ópticos de diferente carga topológica [10]. 

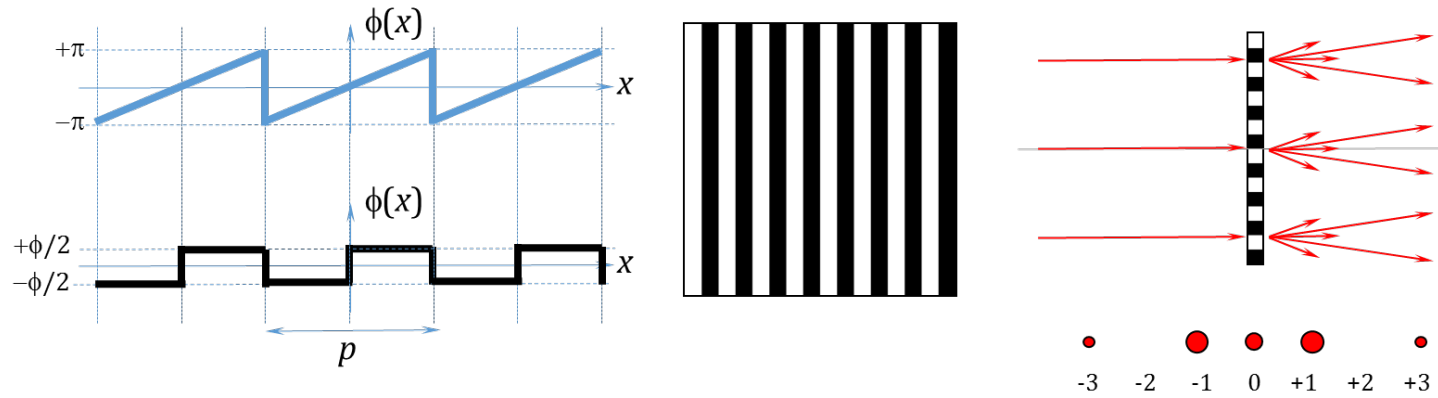

Fig.2. Ilustración de la red de fase binaria.

El patrón de difracción es simétrico, y los órdenes más relevantes son $n=0$, y $n= \pm 1$, con su intensidad relativa dependiente de la diferencia de fase $\phi$ según:

$$
\begin{gathered}
I_{0}=\left|G_{0}\right|^{2}=\frac{1}{2}(1+\cos \phi), \\
I_{ \pm 1}=\left|G_{ \pm 1}\right|^{2}=\frac{2}{\pi^{2}}(1-\cos \phi) .
\end{gathered}
$$

La figura 3 ilustra cómo varían estas intensidades relativas de los órdenes principales con la diferencia de fase $\phi$ entre los dos niveles. La máxima eficiencia de difracción se obtiene cuando $\phi=\pi$. En esta situación los órdenes \pm 1 alcanzan una eficiencia $I_{ \pm 1}=4 / \pi^{2}=40,5 \%$. Se produce entonces un duplicador $(+1,-1)$ con una eficiencia total de nuevo de $\eta=81 \%$.

De las ecuaciones (6) se deriva fácilmente que cuando se cumple que $\cos (\phi)=\left(4-\pi^{2}\right) /\left(4+\pi^{2}\right)$,la intensidad relativa del orden cero es igual a la de los órdenes \pm 1 . Esta situación se da cuando $\phi=0,639 \pi\left(115^{\circ}\right)$ y $\phi=1,361 \pi$ (245을. El valor de la intensidad de cada orden es de $28,8 \%$, de modo que la eficiencia total de este triplicador $(0, \pm 1)$ es $\eta=86 \%$.

La medida de la intensidad de estos órdenes de difracción y su comparación con las curvas teóricas en la figura 3 es un método usual para la determinación de la diferencia de fase de moduladores [14], y lo hemos utilizado en este trabajo para realizar la calibración del modulador LCOS - SLM.

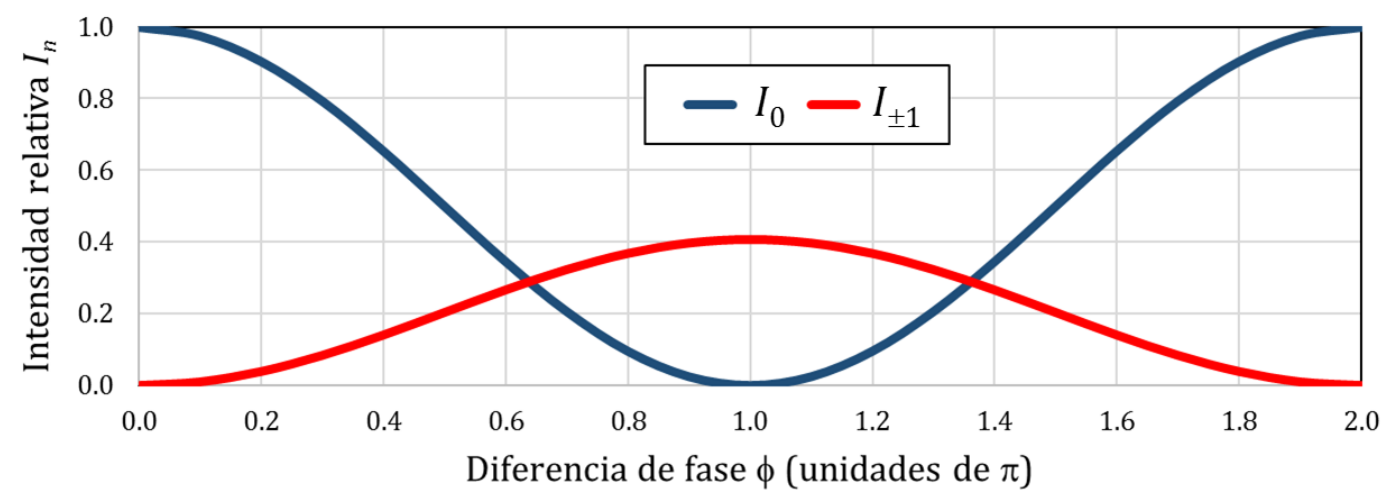

Fig. 3. Evolución de la intensidad de los órdenes de difracción $0, y \pm 1$ en función de la diferencia de fase $\phi$ en una red binaria de fase. La máxima eficiencia de difracción se obtiene para $\phi=180^{\circ}$.

\section{2.c. Redes de Dammann}

Las redes de Dammann están diseñadas para crear patrones de difracción en los que la intensidad se mantiene constante en un número dado de órdenes son iguales. Son redes binarias de fase, con valores de fase de 0 y $\pi$. Se diseñan calculando los puntos de transición $0-\pi$ y $\pi-0$ en el periodo que generan igual intensidad en los órdenes seleccionados [7,8]. Estos puntos de transición se escogen de modo que modo que el desarrollo de la función en la Eq. (1) proporcione coeficientes de Fourier $G_{n}$ con igual intensidad (Eq. (2)), y con el máximo valor posible. No es tanto el objetivo de este trabajo proporcionar la base 
teórica para estos diseños, pues existen estudios que proporcionan en detalle estos puntos de transición para un gran número de diversos casos [9]. En este trabajo, y a pesar de que la estructura pixelada del dispositivo SLM impone restricciones a los puntos de transición que pueden reproducirse, tomamos los valores aportados en dicha referencia [9] y seleccionamos un periodo de la red suficientemente grande de modo que se obtenga una buena aproximación.

Como en los casos anteriores, la generación de la red de Dammann puede interpretarse como una LUT de valores de fase que se aplica a la red de fase lineal, y que modifica el reparto de la energía entre los diversos órdenes de difracción, tal y como ilustra la figura 4 para el caso de la red de Dammann generadora de cinco órdenes iguales $(n=0, \pm 1$ y \pm 2$)$. Según [9], los puntos de transición deben producirse en las posiciones $0,03863,0,39084$ y 0,65552 (donde 1 indica el periodo de la red). Los niveles mostrados como blanco y negro en la figura corresponden a dos niveles de fase con una diferencia relativa de $\pi$ radianes entre ellos. La eficiencia total de difracción de este quintuplicador $(0, \pm 1, \pm 2)$ es igual a la suma de las intensidades relativas de estos cinco órdenes de difracción, y alcanza el valor de $\eta=77 \%$ [9].

Con objeto de realizar una comparación, realizaremos también la red de Dammann que actúa como triplicador (genera tres órdenes $0 \mathrm{y} \pm 1$ de igual intensidad). Según [9], este caso se obtiene con un único punto de transición en la posición 0,73526 . La eficiencia total de difracción teórica de este triplicador $(0, \pm 1)$ alcanza solamente $\eta=66 \%$ [9], sensiblemente menor que la que se puede obtener en el triplicador explicado en la sección anterior, mediante el ajuste de la fase entre los dos niveles de la red.

\section{2.d. El triplicador óptimo de fase}

El último tipo de red de difracción que consideramos es el conocido como triplicador óptimo de fase, cuya derivación teórica fue propuesta por Gori et al en el trabajo [11]. Se trata de una red continua de fase, y supone una red alternativa al diseño de Dammann para el caso con $n=3$ órdenes. Tiene la ventaja de que proporciona una mayor eficiencia de difracción que la red de Dammann.

La expresión matemática de la fase $\phi(x)$ del triplicador óptimo es la siguiente:

$$
\tan (\phi(x))=2 \mu \cos (x)
$$

donde $\mu=1,32859$. La figura 4(b) ilustra esta red de perfil continuo de fase, con una variación total de fase de aproximadamente $0,78 \pi$ radianes. Esto lo hace especialmente interesante para su utilización en moduladores con poca modulación de fase. La eficiencia total de difracción de este triplicador $(0, \pm 1)$ es de $\eta=93 \%$, sensiblemente mejor que la obtenida en los dos ejemplos anteriores de triplicadores.

Como en el caso anterior, la realización de un perfil de fase continuo como el del triplicador óptimo en un modulador pixelado, y por tanto con valores discretos en la modulación espacial de la fase, no puede realizarse de manera exacta. Pero, nuevamente, la elección de un periodo de la red suficientemente grande permite obtener resultados con una buena aproximación. 

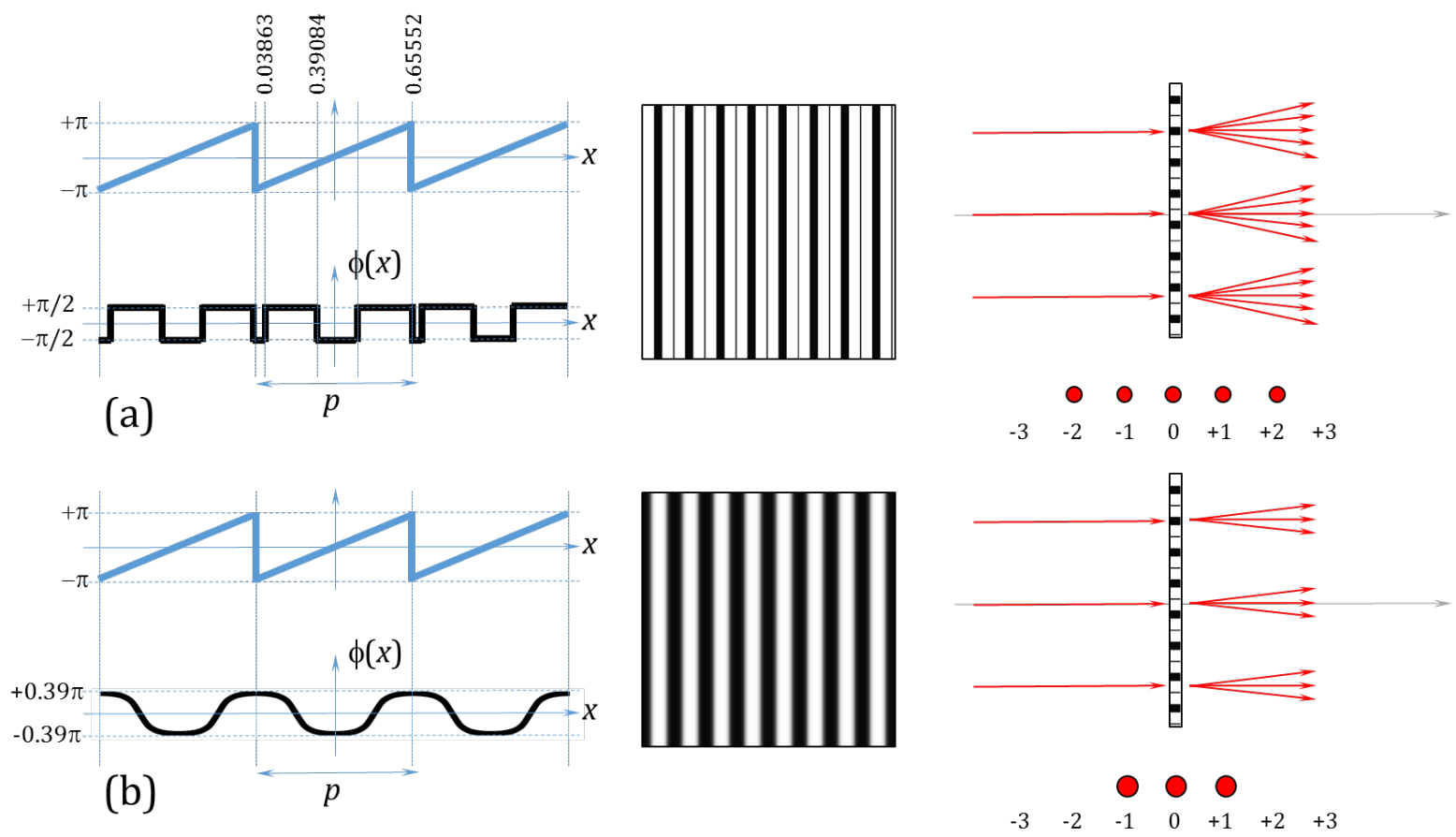

Fig. 4. Ilustración de: (a) la red de Dammann que produce 5 órdenes equi-intensos $(n=0, \pm 1$ y \pm 2$)$, (b) el triplicador óptimo de fase.

\section{Sistema experimental}

La figura 5 muestra un esquema y una fotografía del sistema experimental realizado. Al emplear modulador en reflexión, hemos realizado una geometría óptica de este tipo, en forma de "L", mediante un cubo divisor. De esta forma se consigue incidencia normal sobre el modulador donde se realizarán las redes de difracción.

Se ha empleado un dispositivo LCOS-SLM de la marca Hamamatsu, modelo X10468-01. Los moduladores Hamamatsu de la serie X10468 son SLMs de cristal líquido nemático con alineamiento paralelo, de modo que actúan como SLM puros de fase si la polarización de entrada es paralela al director molecular, que está orientado en dirección horizontal. El dispositivo se controla desde un ordenador mediante interfaz DVI. Las características del modulador son:

- Opera dentro de un rango de longitudes de onda: 400 - $700 \mathrm{~nm}$.

- Número de píxeles: $475.200(792 \times 600)$.

- Tamaño del píxel: $20 \mu \mathrm{m} \times 20 \mu \mathrm{m}$

La modulación óptica del dispositivo se controla mediante el nivel de gris aplicado desde el ordenador de control, que puede tomar valores entre 0 y 255. El fabricante indica que para la longitud de onda de $\lambda=633$ $\mathrm{nm}$ se alcanza una variación lineal de la fase de $2 \pi$ radianes con una variación de 196 niveles de gris. Por ello, empleamos un láser de He-Ne. Mediante un filtro espacial y una lente convergente se obtiene un haz colimado que ilumina la pantalla del modulador LCOS-SLM. El polarizador lineal LP1 se orienta con su eje de transmisión horizontal, de modo que la polarización incidente sea paralela al eje director del modulador. Se añaden al sistema un atenuador con el fin de controlar la intensidad del haz incidente el modulador, y un desfasador de media onda $(\lambda / 2)$, con objeto de controlar la polarización.

La segunda parte del sistema realiza una transformada de Fourier óptica y focaliza el patrón de difracción en una cámara CCD que captura los resultados. Con objeto de poder variar de forma controlada la focal del sistema transformador de Fourier, y por tanto la escala del patrón de difracción, hemos realizado un sistema de dos lentes (L2 y L3), en el que variando la distancia entre las lentes se consigue variar la distancia focal del sistema. Se sitúa también un segundo polarizador lineal (LP2), orientado paralelo a LP1, con objeto de eliminar otros efectos de polarización adicionales que pueden producirse en el cubo divisor. 


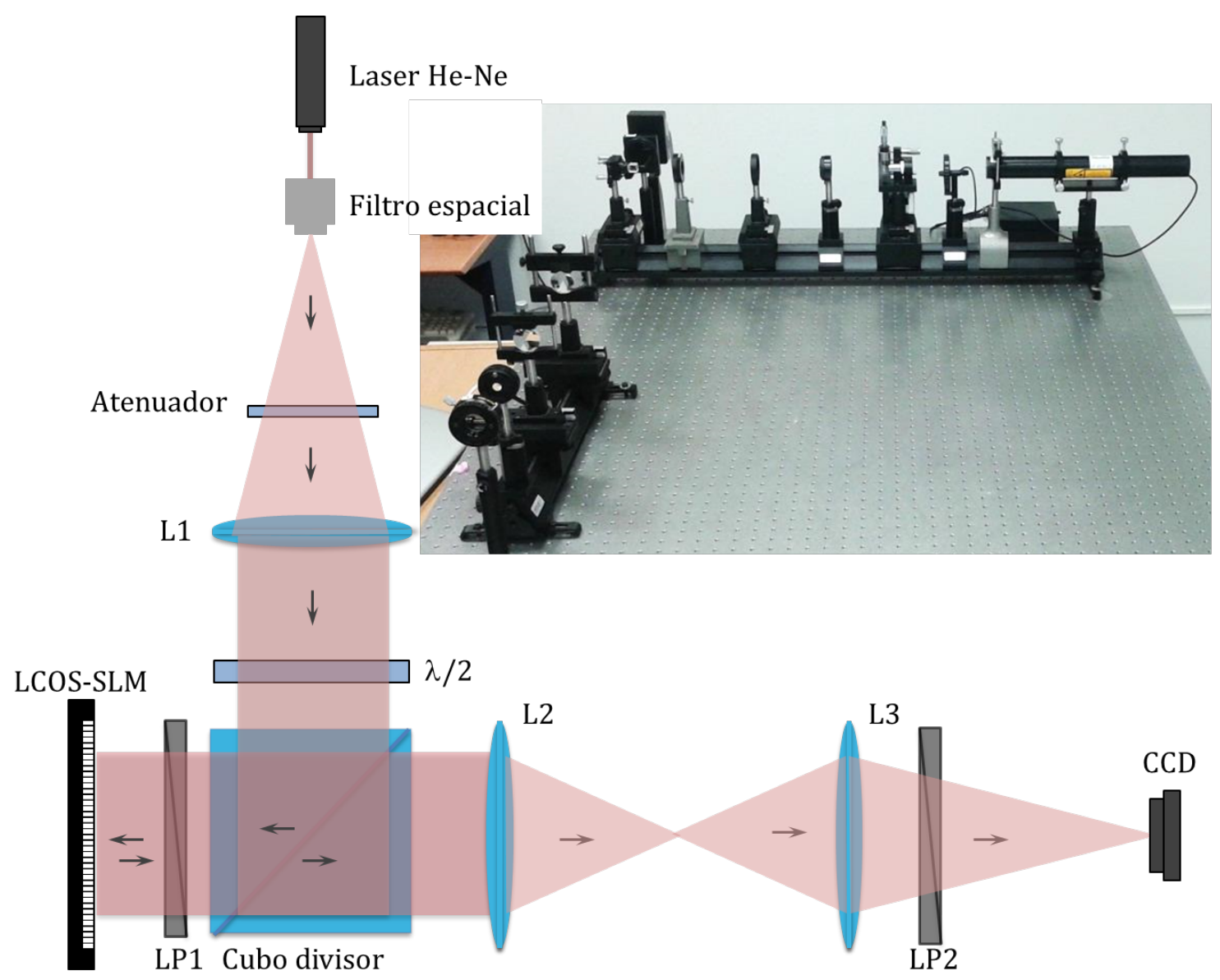

Fig. 5. Esquema del dispositivo experimental. L1, L2 y L3 indican lentes convergentes. LP1 y LP2 indican polarizadores lineales. $\lambda / 2$ indica un desfasador de media onda.

\section{Resultados}

A continuación, presentamos los resultados experimentales obtenidos.

\section{4.a. Redes de fase de perfil binario: calibración del dispositivo}

En primer lugar, consideramos la red binaria de fase, en la que variamos gradualmente la diferencia de fase entre los dos niveles, con objeto de reproducir las predicciones teóricas de la Fig. 3, y de calibrar el dispositivo LCOS-SLM. Para ello realizamos una serie de imágenes de barras verticales binarias, en las cuales un nivel se mantiene fijo en cero (negro), mientras que el otro nivel de gris $(g)$ se varía gradualmente desde cero hasta el valor $g=196$ indicado por el fabricante. La figura 6 muestra los resultados obtenidos. En estos resultados se ha evitado saturar la cámara, con objeto de poder usar los valores relativos como medida de la intensidad relativa entre los órdenes de difracción.

Se observa cómo a medida que aumenta el nivel de gris, la energía del orden cero progresivamente se deriva hacia los órdenes \pm 1 . Para el nivel de gris $g=74$ se alcanza una diferencia de fase que aproxima el valor $\phi=0,639 \pi$, y se obtiene un triplicador $(0, \pm 1)$. Para el nivel de gris $g=98$ se alcanza una diferencia de fase $\phi=\pi$, y se obtiene la extinción del orden cero, y la máxima eficiencia de difracción en los órdenes \pm 1 . A partir de este nivel, la energía en los órdenes \pm 1 comienza a decrecer y a aumentar la de orden cero, de acuerdo a la predicción de la Fig. 3. Para el nivel de gris $g=147$ se alcanza una diferencia de fase que aproxima el valor $\phi=1,361 \pi$, y se obtiene de nuevo un triplicador $(0, \pm 1)$. Para $g=196$ se alcanza la diferencia de fase $\phi=2 \pi$, y se obtiene de nuevo únicamente el orden cero. 

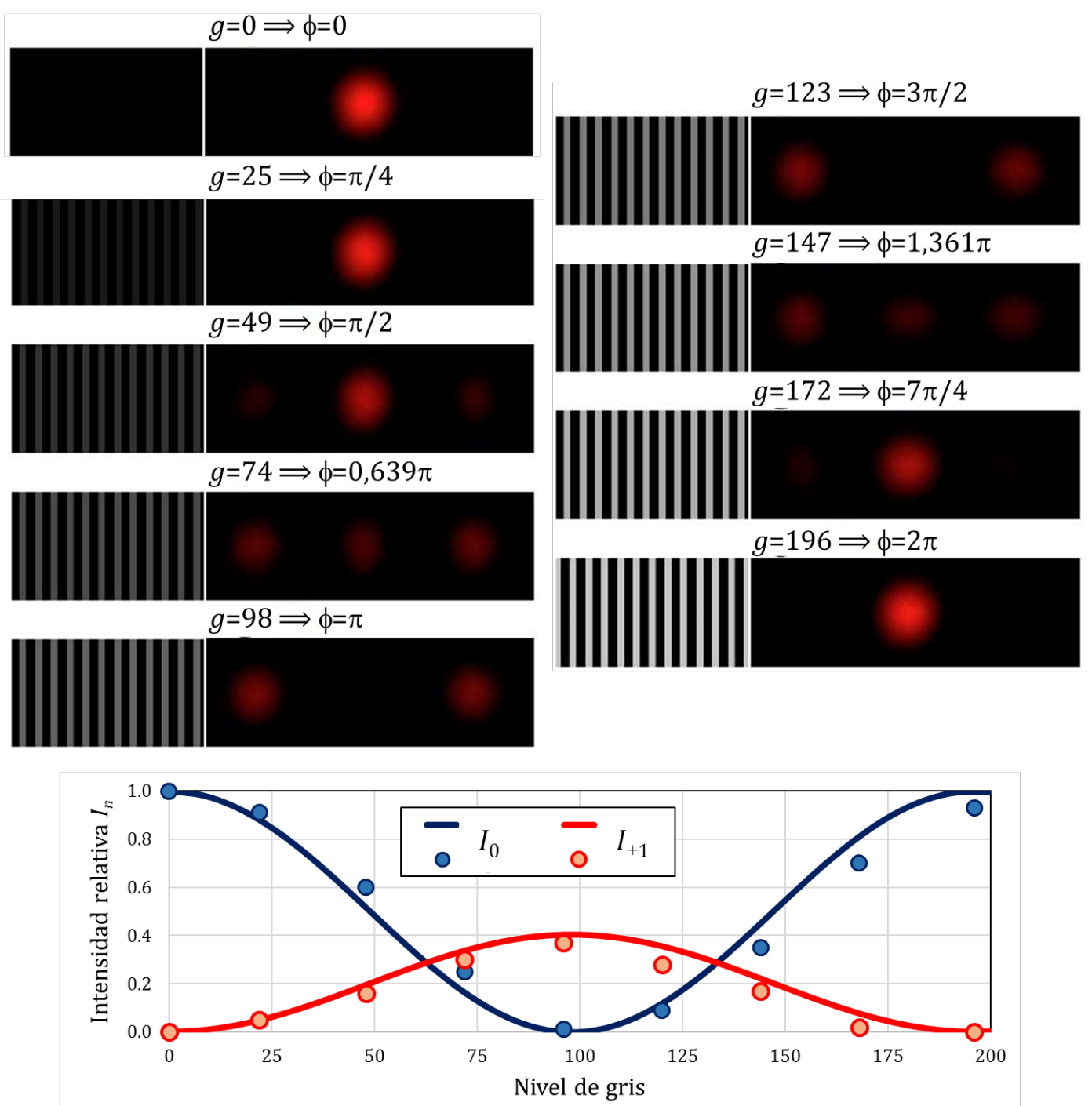

Fig. 6. Resultado experimental de los órdenes de difracción $0, \mathrm{y} \pm 1$ de una red binaria de fase, en función del nivel de gris aplicado. La gráfica inferior muestra los valores normalizados de la intensidad de los órdenes de difracción en comparación con los valores de las ecuaciones (6a) y (6b).

En la gráfica inferior de la figura se comparan los resultados experimentales obtenidos en relación con la diferencia de fase esperada para cada nivel de gris, suponiendo un comportamiento lineal de la fase respecto a $g$. Los valores experimentales de la intensidad (puntos) se han normalizado con respecto al valor medido en el orden cero para $g=0$. Se observa un excelente ajuste, por lo que podemos concluir que este modulador proporciona una respuesta de fase lineal con el nivel de gris. Esta es una ventaja interesante, ya que no es necesario pre-compensar la imagen de niveles de fase diseñada.

\section{4.b. Redes de fase de perfil continuo}

A continuación, hemos realizado redes de fase lineal como las descritas en el apartado 2.a. Para ello se diseñan imágenes con una graduación lineal y periódica del nivel de gris, como las que se muestran en la figura 7. Hemos diseñado dos redes de fase lineal, la que alcanza el valor $\pi$ (cuando el máximo nivel de gris crece hasta alcanzar el nivel $g=98$ ), y la que alcanza el valor $2 \pi$ (cuando el máximo nivel de gris crece hasta alcanzar el nivel $g=98$ ).

Los resultados de difracción de la figura 7 muestran como la energía inicialmente en el orden cero (cuando la imagen en el modulador es uniforme), se difracta totalmente al primer orden de difracción en el segundo caso, o se reparte con igual intensidad (duplicador) entre el orden cero y el orden uno en el 
primer caso. En este caso se observa además como el signo del orden difractado depende del sentido de la pendiente de la red de fase.

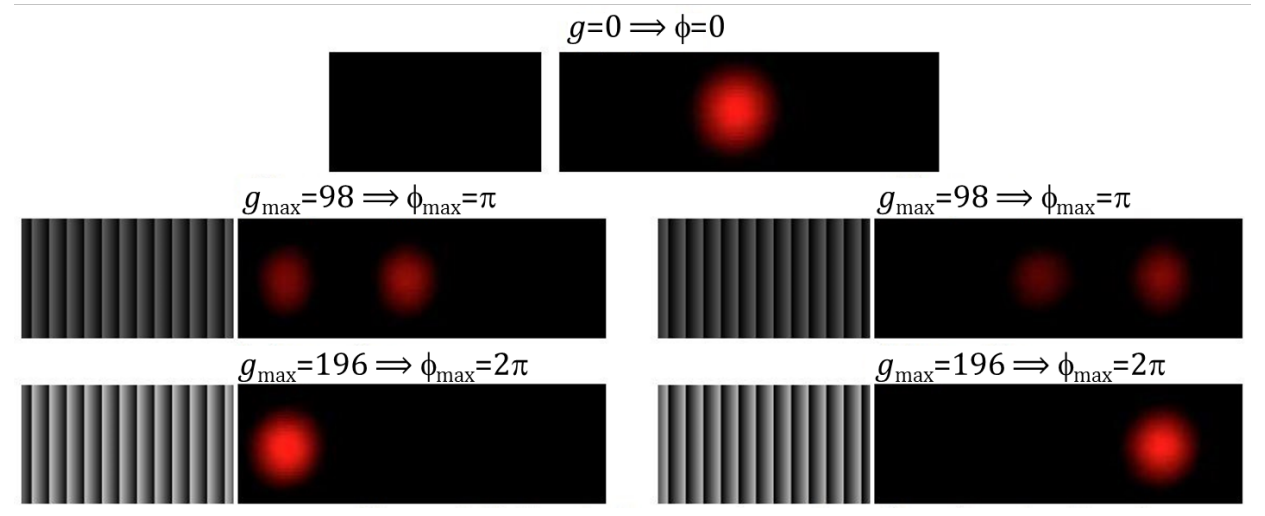

Fig. 7. Resultado experimental de los órdenes de difracción $0, y \pm 1$ de una red de fase lineal para dos casos de la máxima fase.

\section{4.c. Redes de Dammann}

Como tercer ejemplo hemos considerado la realización de redes de Dammann diseñadas para producir $n=5$ órdenes de difracción equi-intensos $(0, \pm 1, \pm 2)$, de acuerdo a los puntos de transición indicados en la Ref. [9]. La figura 8(a) muestra los resultados capturados por la cámara. En este caso, se ha ajustado la distancia entre las lentes L2 y L3, para reducir la escala de la transformada de Fourier. A la izquierda se muestra una muestra de la forma que adopta la red de Dammann, donde blanco y negro representan en este caso los niveles de fase cero y $\pi$ respectivamente. Se observa la correcta generación de los cinco órdenes de difracción.

(a)
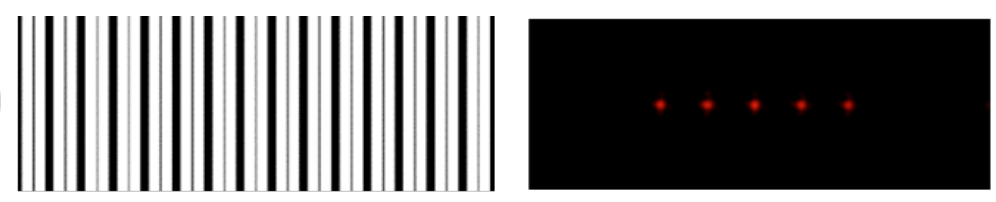

(b)
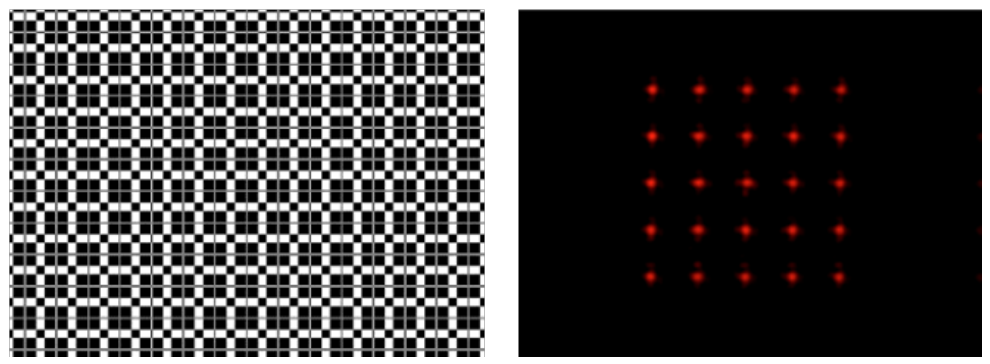

Fig. 8. Resultado experimental de redes de Dammann. (a) Red 1D de $n=5$ órdenes; (b) Red 2D de 5×5 órdenes.

La figura 8(b) muestra la generación de una red de Dammann bidimensional (2D). Esta red se genera simplemente multiplicando la transmisión de la red unidimensional, por una versión de sí misma rotada $90^{\circ}$. Esto es, si la función $g(x)$ representa el perfil de la red unidimensional, la transmisión de la red 2D es simplemente $T(x, y)=g(x) g(y)$. Dado que los niveles de fase $(0, \pi)$ representan valores de transmisión $(+1,-$ $1)$, el resultado de la multiplicación vuelve a ser una función binaria de fase $(0, \pi)$, como la que se observa en el patrón periódico 2D de la figura 8(b). El resultado en el plano de transformada de Fourier es igual a la réplica del patrón de cinco órdenes difractados en dirección vertical sobre cada uno de los órdenes en dirección horizontal, creando así el patrón característico de $5 \times 5$ órdenes que se muestra en la figura.

\section{4.d. Triplicador óptimo de fase}

Como último caso se presenta la realización del triplicador óptimo de fase [11]. Y con objeto de comparar la eficiencia de difracción, se ha realizado la versión equivalente como un triplicador de red de Dammann con $n=3$. El resultado se muestra en la figura 9. A la izquierda se muestran los resultados para el triplicador de Dammann. A la derecha los resultados para el triplicador óptimo. En cada caso se muestra la 
forma que adopta la imagen que codifica la red (fila superior). En el caso de la red Dammann, los niveles blanco y negro corresponden de nuevo a fases cero y $\pi$ respectivamente. En el caso del triplicador óptimo, los niveles de gris representan los niveles de fase, de acuerdo a la teoría expuesta en el apartado 2.d.
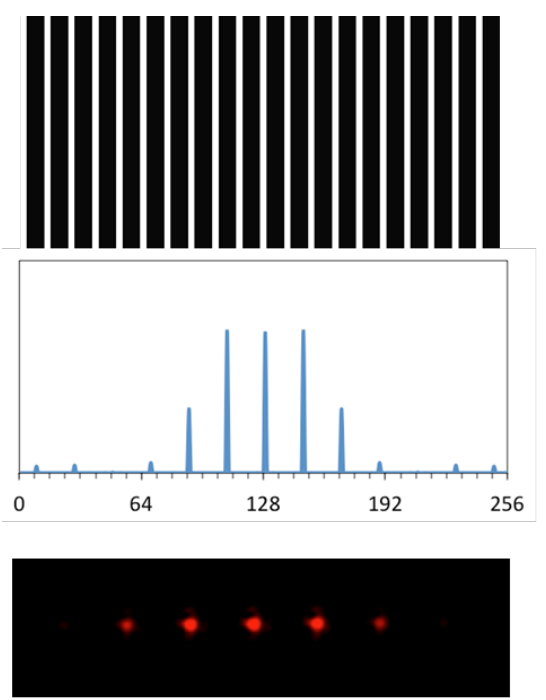

(a)
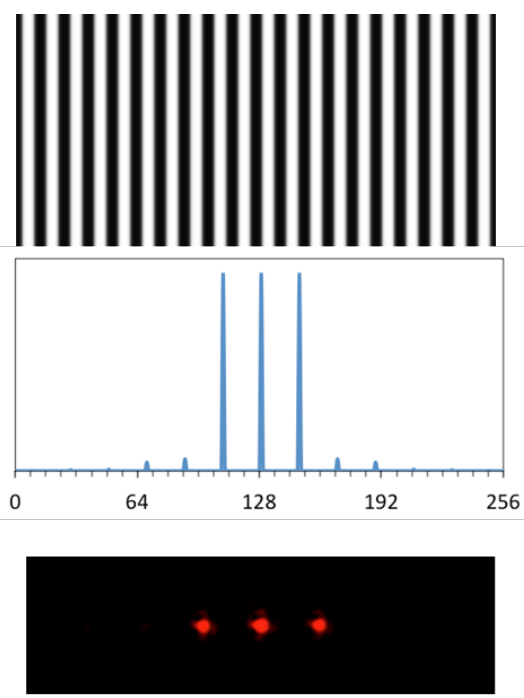

(b)

Fig. 9. (a) Triplicador Dammann 1D de $n=3$ órdenes; (b) Triplicador óptimo de fase.

En ambos casos se ha simulado el perfil del patrón de difracción que se espera obtener. Este perfil se muestra en la segunda fila de la figura. Como se observa, en ambos casos se obtienen tres órdenes de difracción centrales $(0, \pm 1)$ con igual intensidad, pero en el caso del triplicador óptimo son mucho más intensos, minimizando la intensidad de otros órdenes de difracción, mientras que en el triplicador Dammann, otros órdenes de difracción (principalmente los órdenes $n= \pm 2$ ) tienen una intensidad apreciable. Los correspondientes resultados experimentales que se muestran en la última fila verifican esta simulación.

Por último, se ha realizado la versión 2D en ambos casos. Como en el caso anterior de la figura 8(b), la versión 2D se obtiene multiplicando la transmisión compleja de la red 1D por su versión rotada $90^{\circ}$. El resultado en ambos casos es una red bidimensional como las que se muestran en la figura 10. Nuevamente, en el caso de la red Dammann, los valores de blanco y negro representan valores de fase cero y $\pi$ respectivamente, mientras que en la red 2D basada en el diseño óptimo, los niveles de gris corresponden a los niveles continuos de fase.

Los resultados experimentales mostrados en la figura 10 corroboran la generación de una matriz de $3 \times 3$ órdenes de igual intensidad en la parte central con ambas redes. Sin embargo, se muestra claramente la ventaja en términos de eficiencia del triplicador óptimo, que produce los nueve haces de luz centrales mucho más intensos, y reduce en gran medida los otros órdenes de difracción colaterales que se observan con el diseño de Dammann.

\section{Conclusiones}

En resumen, se ha presentado un conjunto de experiencias simples empleando un modulador espacial de luz de cristal líquido para la evaluación de diversos perfiles de redes de difracción de fase: redes de fase binaria simples, redes de fase lineal, redes de Dammann y el triplicador óptimo de fase. Se ha mostrado que el SLM, a pesar de su estructura pixelada, es capaz de reproducir con una buena aproximación perfiles de fase binarios y continuos como éstos, gracias a la elección de un periodo de las redes suficientemente grande, y por tanto permite un análisis más simple apropiado para esta primera aproximación a esta temática. No obstante, se considera como un interesante estudio adicional evaluar los efectos del muestreado espacial y de la cuantización de los niveles de fase que ocurre cuando los periodos de la red se eligen más pequeños. 


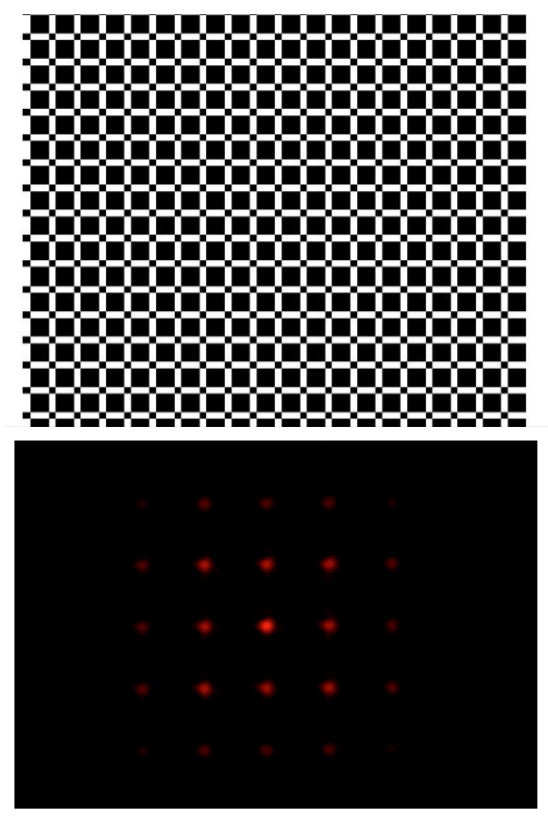

(a)
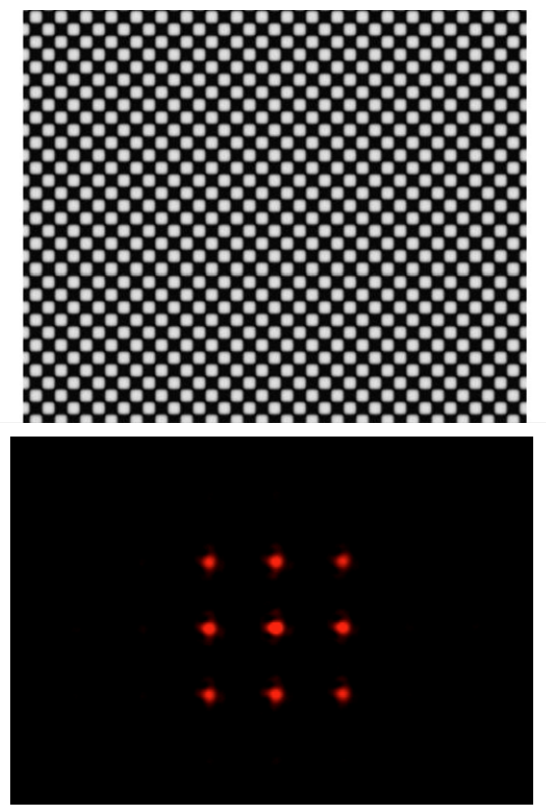

(b)

Fig. 10. (a) Red Dammann 2D de 3×3 órdenes; (b) Red 2D de 3×3 órdenes basada en el diseño de triplicador óptimo.

La realización práctica de este conjunto de diseños teóricos y su realización experimental es un interesante ejercicio práctico, muy útil para la introducción de estos aspectos de la Óptica, tanto a nivel educativo como de iniciación a la investigación en el campo de la óptica difractiva con dispositivos SLM. Puede combinarse con otros tipos de demostraciones prácticas de los dispositivos LCD [15] como una potente herramienta avanzada para la docencia y para la introducción a la investigación en óptica.

Este trabajo se ha realizado en el marco de un proyecto final de carrera en la Universidad Miguel Hernández de Elche [13].

\section{Agradecimientos}

Se agradece el apoyo del Ministerio de Economía y Competitividad a través del proyecto Ref.: FIS201566328-C3-3-R. 